\title{
Huge Variety of Nuclides That Arise in the LENR Processes: Attempt at Explanation
}

\author{
Alexander G. Parkhomov'1, Ekaterina 0. Belousova² \\ ${ }^{1}$ Russian Academy of Natural Sciences, Moscow, Russia \\ ${ }^{2}$ Institute of Experimental Mineralogy, Russian Academy of Sciences, Moscow, Russia \\ Email: alexparh@mail.ru
}

How to cite this paper: Parkhomov, A.G. and Belousova, E.O. (2022) Huge Variety of Nuclides That Arise in the LENR Processes: Attempt at Explanation. Journal of Modern Physics, 13, 274-284. https://doi.org/10.4236/jmp.2022.133019

Received: January 29, 2022

Accepted: March 5, 2022

Published: March 8, 2022

Copyright (c) 2022 by author(s) and Scientific Research Publishing Inc. This work is licensed under the Creative Commons Attribution International License (CC BY 4.0).

http://creativecommons.org/licenses/by/4.0/ (c) (i) Open Access

\begin{abstract}
Researches in the field of low-energy nuclear reactions (LENR) have shown a wide variety of manifestations of these phenomena. They appear in metals with hydrogen dissolved in them, in plasma, in gas discharge, in electrolysis, and even in biological systems. In addition to energy release, which far exceeds the capabilities of chemical reactions, LENR is characterized by a huge variety of emerging chemical elements. This report provides examples of appearance of many initially missing elements in different LENR installations. For example, in the nickel-hydrogen LENR reactor created in our laboratory, which worked for 7 months, $\mathrm{Ca}, \mathrm{V}, \mathrm{Ti}, \mathrm{Mn}, \mathrm{Fe}, \mathrm{Co}, \mathrm{Cu}, \mathrm{Zn}, \mathrm{Ga}, \mathrm{Ba}, \mathrm{Sr}, \mathrm{Yb}, \mathrm{Hf}$ were found. Moreover, new elements were found not only in the "fuel" but also in the surrounding matter. The huge variety of chemical elements that arise can be explained by the fact that in the processes of LENR, the interaction covers several atoms at once. The article discusses approaches to explaining the phenomena discovered in the process of LENR researches.
\end{abstract}

\section{Keywords}

Nuclear Transmutations, LENR, Coulomb Barrier, Multicore

Transformations, Hot Metals, Particles Collisions, Weak Nuclear Interaction, Neutrino, Low Energies

\section{Introduction}

This article gives special attention to an important feature of LENR: huge variety of emerging new nuclides. In the experiments of M. Fleischmann and S. Pons, which began intensive research in the field of LENR, tritium and helium were presumably formed during the fusion of deuterium nuclei. But soon it was discovered that cold nuclear transformations are not limited only to the fusion of 
light nuclides. This paper provides a few examples of the various nuclear transformations found in various numerous experiments.

\section{Examples of Huge Variety of Emerging New Nuclides}

Experiments by I.B. Savvatimova, A.B. Karabut and Y.R. Kucherov [1] [2] began almost immediately after the publication by M. Fleischmann and S. Pons. In these experiments, the effect of a glow discharge in a medium of deuterium and other gases on palladium and other metals was investigated. Release of excess energy and many initially absent chemical elements were found. For instance, Sc, Ti, V, Ag, Cd, In, P, Cl, Br, Ge, As, Kr, Sr, Y, Ru, Xe were found in Pd. In some elements an unusual ratio of isotopes was found. In addition, gamma and neutron radiation was detected, as well as the appearance of radioactivity in the irradiated targets. However, detected radiation intensity and radioactivity were very weak. Thus, already in these early studies, many important properties of LENR were identified.

At the same time, in Magnitogorsk State Technical University, Anatoly Vachaev created an installation called "Energoniva" [3]. In this installation, water flowed between tubular electrodes, between which a special type of electric discharge was created. As a result, many elements that were initially absent appeared in the water, from lithium to lead. Most of all, iron appeared. In addition, heavy and superheavy water accumulated in the water, i.e. deuterium and tritium appeared. Vachaev's installation was reproduced in several laboratories. The appearance of many initially absent nuclides occurred everywhere.

Many initially absent nuclides from boron to lead were recorded in the wellknown experiments of Leonid Urutskoev with exploding metal foils [4]. A change in the isotopic composition of titanium after an electric explosion was detected. In addition, in these experiments, "strange radiation" was detected, which formed unusual tracks in the photo emulsion and on the surfaces of various detectors [4] [5].

In the experiments of Ubaldo Mastromatteo [6] and Jean-Paul Biberian [7], palladium foils were subjected to prolonged laser irradiation in an atmosphere of hydrogen and deuterium. In the field of irradiation, many new chemical elements (N, O, Na, Mg, $\mathrm{Al}, \mathrm{S}, \mathrm{Ca}, \mathrm{Fe}, \mathrm{Ni}, \mathrm{Zn}, \mathrm{Mo}$ ) and a significant decrease in the content of palladium were detected.

In our laboratory, various substances were exposed to incandescent lamps [8] [9]. Release of excess heat and changes in the elemental composition in the substances surrounding the lamp were detected. In one of the experiments, the tube-shaped incandescent lamp was wrapped in a lead-tin alloy ribbon. In order to prevent the alloy from melting, running water was used. Many elements ranging from lithium to bismuth were reliably detected.

It is important to note the following. Many attempts to explain LENR include hydrogen or deuterium as a necessary element of the process. In the lead-tin alloy ribbon experiment, nuclear transmutations occur in the complete absence of 
hydrogen. This indicates the need for approaches to explain LENR that do not require the mandatory presence of hydrogen.

Many nickel-hydrogen reactors have been tested in our laboratory [10]. In some of them, elemental and isotopic composition of both the nickel-hydrogen core and the surrounding matter was analyzed. Many initially absent elements were always detected, not only in the core, but also in the surrounding matter.

One of our reactors (M7) ran continuously for 7 months, generating up to 1000 watts of excess power [11]. Figure 1 and Figure 2 show what new elements appeared in different parts of the reactor after the end of its operation. New elements (K, Ca, V, Cr, Ti, Mn, Fe, Zn, Ga, Sr, Ba, Yb, Hf) appeared in all parts of the reactor located near the core, even in the outer ceramic tube. Analysis was made of the isotopic composition of nickel, which, along with hydrogen, was considered as "fuel". Contrary to expectations, even after a long stay in a working reactor, it almost did not change. The isotopic composition of nickel remained

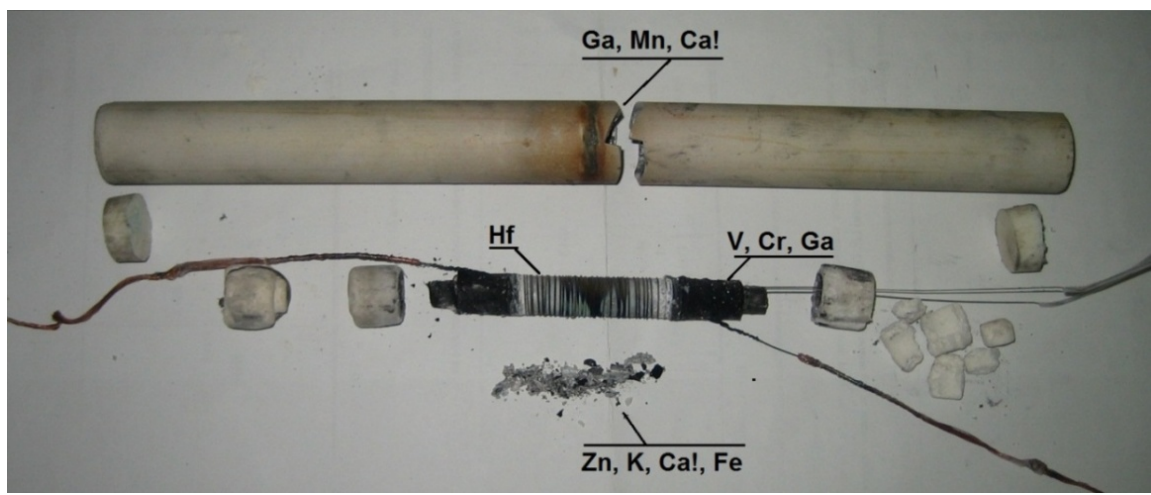

Figure 1. M7 reactor after shutdown. Many elements that had been initially all but absent in the fuel were found in the fuel and in the reactor structure and calcium in especially significant quantities. In the inner ceramic tube the calcium content reached $23 \%$ with an initial content of about $1 \%$.

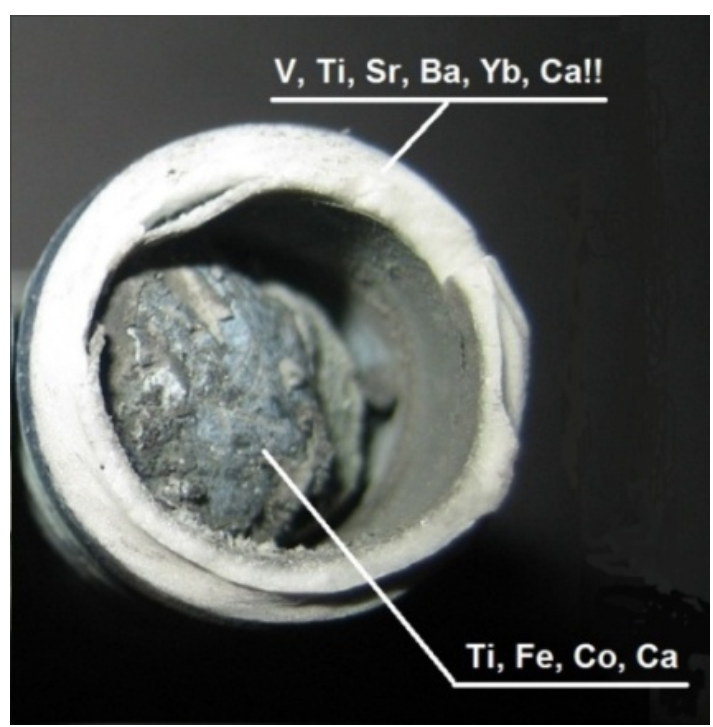

Figure 2. Cross section of M7 reactor inner tube. 
almost unchanged in our other nickel-hydrogen reactors. This indicates that LENR processes (heat generation, transmutations) occur not so much in the hot nickel-hydrogen core, but in surrounding matter.

\section{Important features of LENR}

Numerous diverse experiments allow us to highlight a number of important features of LENR:

1) huge variety of nuclides appears not only in the "fuel", but also in surrounding matter, and, in general, nuclides that do not have radioactivity appear;

2) energy is released far beyond the capabilities of chemical reactions;

3) neutrons and gamma radiation are emitted during the LENR process. However, the radiation intensity is many orders of magnitude lower than in "normal" nuclear reactions;

4) a dense medium (solid, liquid, dense plasma) is required;

5) unusual tracks appear near the reactors.

\section{Attempts to Explain the LENR Phenomenon}

Many attempts have been made to explain the LENR phenomenon. Numerous hypotheses can be divided into several groups:

- proton (deuteron) overcomes the "Coulomb barrier" and merges with the nucleus;

- proton turns into a neutron, for which there is no "coulomb barrier". This neutron generates a chain of nuclear transformations;

- LENR processes involve a catalyst;

- An atom enters a compact state with the release of high energy. In addition, an atom in a compact state overcomes the "Coulomb barrier" more easily.

But so far, it has not been possible to explain all the features of LENR, or at least some of them without contradicting the rest. Basically, the search went in the direction of finding ways to overcome the "Coulomb barrier", which prevents a sufficiently close approach of the nuclei. An example of this approach is the well-known Widom-Larsen theory [12], which allows possibility of converting protons into neutrons. Resulting neutrons react with the nuclei of surrounding matter, forming new chemical elements. However, in reality, experiments did not detect the intense radiation of neutrons and hard gamma rays, which inevitably occur when neutrons are captured by nuclei. This alone refutes this type of hypothesis. Similar inconsistencies are typical for other approaches. It should be emphasized that none of the above approaches provide an explanation for the huge variety of nuclides that arise.

\section{Assumption of Energy-Efficient Rearrangements of Nucleons Located in More Atoms}

The problem of the appearance of huge variety of emerging nuclides can be solved by the assumption that they appear as a result of energy-efficient rear- 
rangements of nucleons located in two or more atoms [13] [14]. To do this, they must be exposed to action that covers several atoms at the same time. Indeed, if one assumes the multi-nucleated nature of the transformations, the appearance of numerous diverse nuclides becomes possible. Papers [15] [16] describe results of computer calculations of the simplest variant of multicore transformations: exothermic transformations of one or two stable nucleons into one or two stable nucleons with or without the participation of electrons. A computer calculation revealed more than a million variants in this simplest type of multicore transformation.

Possible nuclear transformations in corundum $\left(\mathrm{Al}_{2} \mathrm{O}_{3}\right)$ are shown below as an example. Corundum is substance that has been widely used in our reactors. Even when considering the interactions of only two aluminum nuclei, many new helium-to-iron nuclides can arise:

$$
\begin{aligned}
& { }^{27} \mathrm{Al}+{ }^{27} \mathrm{Al} \rightarrow{ }^{54} \mathrm{Fe}+21.242 \mathrm{MeV} \quad{ }^{27} \mathrm{Al}+{ }^{27} \mathrm{Al} \rightarrow{ }^{50} \mathrm{Cr}+{ }^{4} \mathrm{He}+13.428 \mathrm{MeV} \\
& { }^{27} \mathrm{Al}+{ }^{27} \mathrm{Al} \rightarrow{ }^{42} \mathrm{Ca}+{ }^{12} \mathrm{C}+4.137 \mathrm{MeV}{ }^{27} \mathrm{Al}+{ }^{27} \mathrm{Al} \rightarrow{ }^{38} \mathrm{Ar}+{ }^{16} \mathrm{O}+5.058 \mathrm{MeV} \\
& { }^{27} \mathrm{Al}+{ }^{27} \mathrm{Al} \rightarrow{ }^{34} \mathrm{~S}+{ }^{20} \mathrm{Ne}+5.058 \mathrm{MeV}{ }^{27} \mathrm{Al}+{ }^{27} \mathrm{Al} \rightarrow{ }^{30} \mathrm{Si}+{ }^{24} \mathrm{Mg}+3.971 \mathrm{MeV} \\
& { }^{27} \mathrm{Al}+{ }^{27} \mathrm{Al} \rightarrow{ }^{29} \mathrm{Si}+{ }^{25} \mathrm{Mg}+0.688 \mathrm{MeV}{ }^{27} \mathrm{Al}+{ }^{27} \mathrm{Al} \rightarrow{ }^{28} \mathrm{Si}+{ }^{26} \mathrm{Mg}+3.308 \mathrm{MeV}
\end{aligned}
$$

Additional nuclides can arise from interactions of two oxygen nuclei and nuclei of oxygen and aluminum:

$$
\begin{array}{ll}
{ }^{16} \mathrm{O}+{ }^{16} \mathrm{O} \rightarrow{ }^{32} \mathrm{~S}+16.539 \mathrm{MeV} & { }^{16} \mathrm{O}+{ }^{16} \mathrm{O} \rightarrow{ }^{31} \mathrm{P}+{ }^{1} \mathrm{H}+7.673 \mathrm{MeV} \\
{ }^{16} \mathrm{O}+{ }^{16} \mathrm{O} \rightarrow{ }^{28} \mathrm{Si}+{ }^{4} \mathrm{He}+9.594 \mathrm{MeV} & { }^{16} \mathrm{O}+{ }^{27} \mathrm{Al} \rightarrow{ }^{42} \mathrm{Ca}+{ }^{1} \mathrm{H}+9.312 \mathrm{MeV} \\
{ }^{16} \mathrm{O}+{ }^{27} \mathrm{Al} \rightarrow{ }^{39} \mathrm{~K}+{ }^{4} \mathrm{He}+9.438 \mathrm{MeV} & { }^{16} \mathrm{O}+{ }^{27} \mathrm{Al} \rightarrow{ }^{31} \mathrm{P}+{ }^{12} \mathrm{C}+2.502 \mathrm{MeV}
\end{array}
$$

Thus, in corundum $\mathrm{H}, \mathrm{He}, \mathrm{C}, \mathrm{O}, \mathrm{Ne}, \mathrm{S}, \mathrm{Ar}, \mathrm{Mg}, \mathrm{Si}, \mathrm{P}, \mathrm{Ca}, \mathrm{Cr}, \mathrm{Fe}$ may appear. Many of these new elements were discovered by analyzing the corundum tubes that worked in our reactors.

It should be noted that such transformations cannot occur through the channel of strong interactions due to the "Coulomb barrier". But nothing prevents, in principle, such transformations from occurring through the channel of weak nuclear interactions. In this case, a neutrino or antineutrino must be present in the equations of nuclear reactions.

It is important to note that transformations involving an ensemble of a large number of nuclei occur mainly without the formation of the radioactive nuclides and excited nuclei that cause gamma radiation. This is due to the fact that in a system of a large number of interacting nuclei, there are many options for the formation of products with stable and unstable isotopes, with excited and unexcited nuclei. In accordance with the Ziegler principle, a non-equilibrium system develops in such a way as to maximize entropy production under given external constraints [17]. Entropy production in the formation of stable and unexcited nuclides is higher than in the formation of unstable and excited ones, so their formation is more likely.

As already mentioned, for the implementation of multi-core interactions, a mechanism covering several atoms is necessary. Here are a few hypothetical 
ways to implement multinucleated transformations:

- Magnetic monopoles [4] [5] [17] [18] [19] [20] [21];

- "Capsules" with transatoms [22];

- Magneto-toro-electric clusters [23];

- High density Charge Clusters [24];

- Ultra-low-energy neutrinos [8] [25].

Let us consider the last of the listed approaches, as it allows one without contradiction to explain the main features of LENR and without involving "new entities" devoid of experimental confirmation.

\section{Hypothesis of Low-Energy Neutrinos Participation in Nuclear Transformations}

Theoretical works on the interaction of neutrinos with matter at very low energies give an extremely low probability of interaction [26] [27]. But a number of experiments indicate that, in fact, neutrinos at low energies interact with matter quite noticeably [28] [29].

It is important to note that approaches mastered by high-energy neutrino physics are unsuitable for very low-energy neutrinos (of the order of $1 \mathrm{eV}$ and below). The difference in energy is about the same as between that of gamma quanta and light photons. Photons manifest themselves in a completely different and much more diverse way than gamma quanta. Apparently, neutrinos at very low energies also have many important but unexplored properties. But something is quite clear. The large de Broglie wavelength, which is many times greater than the interatomic distances, leads to the fact that the interaction covers a huge number of atoms. Of very low energy neutrinos interact with matter macroscopically, i.e. they are refracted, reflected at the boundaries of media, scattered on inhomogeneities. Streams of such neutrinos experience interference and diffraction. In these processes, there is practically no energy exchange between neutrinos and matter.

In addition, neutrinos work in nuclear transformations involving the weak interaction. Unlike other types of interactions, the role of the weak nuclear interaction is not in attraction or repulsion, but in the transformation of neutrons into protons or protons into neutrons with the participation of electrons or positrons and antineutrinos and neutrinos. At the same time, in addition to fulfilling the laws of conservation of energy, momentum, angular momentum, baryon and electric charge, the lepton charge must be preserved. Neutrinos or antineutrinos act as a kind of key that resolves nuclear reactions of weak interaction. In addition, they can contribute additional energy. For example, the well-known nuclear reaction $v+\mathrm{p} \rightarrow \mathrm{e}^{+}+\mathrm{n}$ is possible when the neutrino energy is greater than $1806 \mathrm{keV}$. But if the energy balance of the nuclear conversion is positive, no additional energy is required, only the presence of neutrinos. For example, the reverse beta decay of $v+{ }^{60} \mathrm{Co} \rightarrow{ }^{60} \mathrm{Ni}+\mathrm{e}^{-}+2.823 \mathrm{MeV}$ can occur at an arbitrarily low neutrino energy. However, such nuclei have spontaneous beta radioactivity, 
therefore this reaction must be observed against the background of spontaneous decays. A noticeable effect can be at a sufficiently high neutrino flux density. This is achieved, for example, in the focus of a parabolic mirror in which a small beta source is located [28] [29]. When scanning the celestial sphere, bursts of ${ }^{60} \mathrm{Co}$ activity were observed, many times exceeding the background of spontaneous radioactivity (sometimes by 1000 times). It is assumed that the increase in the decay rate of the beta source is caused by concentrated flows of very low-energy neutrinos-the dark matter component.

As noted above, a lot of nuclear transformations are, in principle, possible when two or more nuclei are converted into two or more other nuclei with a positive energy balance [8] [15] [16]. The Coulomb barrier does not allow the nuclei to get close enough for the strong nuclear interaction to work. But in the presence of a neutrino that "includes" the weak interaction covering both nuclei, nothing prevents the reactions from occurring, for example:

$$
\begin{gathered}
v+{ }^{27} \mathrm{Al}+{ }^{27} \mathrm{Al}+\mathrm{e}^{-} \rightarrow{ }^{53} \mathrm{Cr}+{ }^{1} \mathrm{H}+13.594 \mathrm{MeV} \\
v+{ }^{27} \mathrm{Al}+{ }^{27} \mathrm{Al} \rightarrow{ }^{50} \mathrm{Cr}+{ }^{4} \mathrm{He}+13.428 \mathrm{MeV}+v^{\prime}
\end{gathered}
$$

The neutrino on the left side of the equations can have an arbitrarily low energy. The neutrino on the right side of the second equation has a high energy and flies away, carrying away a significant part of the released energy.

Currently, there is no exact data on the rest mass of the electron neutrino (antineutrino). Direct experiments show that it is less than $0.8 \mathrm{eV}$ [30]. Estimates based on astronomical observations give a mass value less than $0.28 \mathrm{eV}$ [31]. Studies of neutrino oscillations indicate a neutrino mass value less than $0.05 \mathrm{eV}$ [32]. If it is true that the mass of an electron neutrino and an antineutrino is so small, then neutrino-antineutrino pairs can be formed as a result of inelastic collisions of particles of matter (electrons, ions, neutral atoms) during their thermal motion. The energy of colliding particles must be more than twice the mass of the particles being born. Figure 3 shows the fraction of particles of matter with thermal motion energy greater than 0.1 and $0.5 \mathrm{eV}$ at temperatures up to $10000^{\circ} \mathrm{C}$ [8] [25]. It can be seen that the formation of neutrino-antineutrino pairs is possible in matter at a temperature above a threshold, which depends on the mass of neutrinos and antineutrinos (about $100^{\circ} \mathrm{C}$ if the mass is $0.05 \mathrm{eV}$ and about $1000^{\circ} \mathrm{C}$ if the mass is $0.25 \mathrm{eV}$ ). Note that the production of excess heat in nickel-hydrogen LENR reactors requires heating to a temperature of at least $1100^{\circ} \mathrm{C}$ [10] [11].

Especially often, electrons collide with atoms in metals: in $1 \mathrm{~cm}^{3}$, there are about $10^{36}$ collisions per second [8] [25]. Such a high frequency of collisions leads to the appearance of huge number of neutrinos and antineutrinos, even with a very small probability of their formation. Resulting neutrinos and antineutrinos can interact with the nuclei of surrounding matter. In addition, neutrinos and antineutrinos can appear from light photons, since their energy is sufficient to form a pair of neutrinos-antineutrinos. 


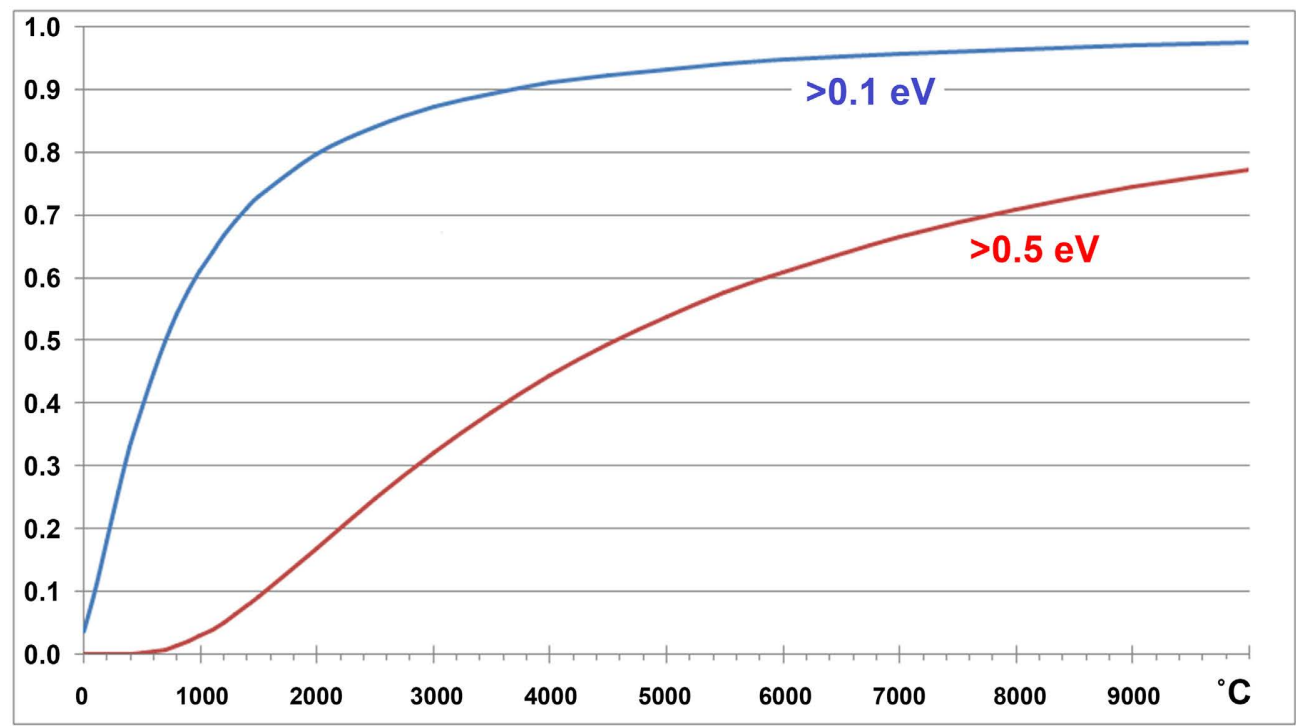

Figure 3. Fraction of matter particles with a thermal motion energy greater than 0.1 and $0.5 \mathrm{eV}$.

It is important to note that de Broglie wavelength $\lambda=h / p$ ( $h$ : Planck constant, $p$ : momentum) of emerging neutrinos and antineutrinos is about 1 micron. This means that region of interaction covers a huge number of atoms, and this makes transformations involving many atoms and nuclei possible, as a result of which even unlikely processes become significant [28] [29] [33].

Figure 4 shows dependence of the de Broglie wavelength, i.e., the size of the interaction region, on neutrino or antineutrino energy. It can be seen that at energies of the order of $10 \mathrm{keV}$ and higher, only one nucleus can be covered by the interaction. At energies of several $\mathrm{keV}$, the interaction already covers several atoms. And neutrinos and antineutrinos, which arise from thermal collisions of atoms of matter with an energy of less than $1 \mathrm{eV}$, cover many billions of atoms by their interaction.

Here are some examples of possible nuclear transformations of two nuclei, electron and neutrino or antineutrino. In nickel and in nickel with participation of hydrogen, iron, cobalt, copper, and helium may appear:

$$
\begin{gathered}
v^{\sim}+{ }^{58} \mathrm{Ni}+{ }^{61} \mathrm{Ni}+\mathrm{e}^{-} \rightarrow{ }^{63} \mathrm{Cu}+{ }^{56} \mathrm{Fe}+1.736 \mathrm{MeV} \\
v^{\sim}+{ }^{58} \mathrm{Ni}+{ }^{64} \mathrm{Ni}+\mathrm{e}^{-} \rightarrow{ }^{65} \mathrm{Cu}+{ }^{57} \mathrm{Fe}+0.113 \mathrm{MeV} \\
v^{\sim}+{ }^{58} \mathrm{Ni}+{ }^{61} \mathrm{Ni}+\mathrm{e}^{-} \rightarrow{ }^{59} \mathrm{Co}+{ }^{60} \mathrm{Ni}+2.252 \mathrm{MeV} \\
v^{\sim}+{ }^{60} \mathrm{Ni}+{ }^{61} \mathrm{Ni}+\mathrm{e}^{-} \rightarrow{ }^{59} \mathrm{Co}+{ }^{62} \mathrm{Ni}+0.276 \mathrm{MeV} \\
v+{ }^{61} \mathrm{Ni}+{ }^{64} \mathrm{Ni} \rightarrow{ }^{65} \mathrm{Cu}+{ }^{60} \mathrm{Ni}+\mathrm{e}^{-}+0.409 \mathrm{MeV} \\
v+{ }^{61} \mathrm{Ni}+{ }^{64} \mathrm{Ni} \rightarrow{ }^{63} \mathrm{Cu}+{ }^{62} \mathrm{Ni}+\mathrm{e}^{-}+0.995 \mathrm{MeV} \\
v^{\sim}+{ }^{60} \mathrm{Ni}+{ }^{1} \mathrm{H}+\mathrm{e}^{-} \rightarrow{ }^{61} \mathrm{Ni}+7.041 \mathrm{MeV} \\
v^{\sim}+{ }^{61} \mathrm{Ni}+{ }^{1} \mathrm{H}+\mathrm{e}^{-} \rightarrow{ }^{62} \mathrm{Ni}+9.808 \mathrm{MeV} \\
v^{\sim}+{ }^{60} \mathrm{Ni}+{ }^{1} \mathrm{H}+\mathrm{e}^{-} \rightarrow{ }^{4} \mathrm{He}+{ }^{57} \mathrm{Fe}+0.569 \mathrm{MeV} \\
v^{\sim}+{ }^{61} \mathrm{Ni}+{ }^{1} \mathrm{H}+\mathrm{e}^{-} \rightarrow{ }^{4} \mathrm{He}+{ }^{58} \mathrm{Fe}+2.794 \mathrm{MeV} \\
v+{ }^{61} \mathrm{Ni}+{ }^{1} \mathrm{H} \rightarrow{ }^{4} \mathrm{He}+{ }^{58} \mathrm{Ni}+\mathrm{e}^{-}+0.865 \mathrm{MeV} \\
v+{ }^{64} \mathrm{Ni}+{ }^{1} \mathrm{H} \rightarrow{ }^{4} \mathrm{He}+{ }^{61} \mathrm{Ni}+\mathrm{e}^{-}+1.98 \mathrm{MeV}
\end{gathered}
$$




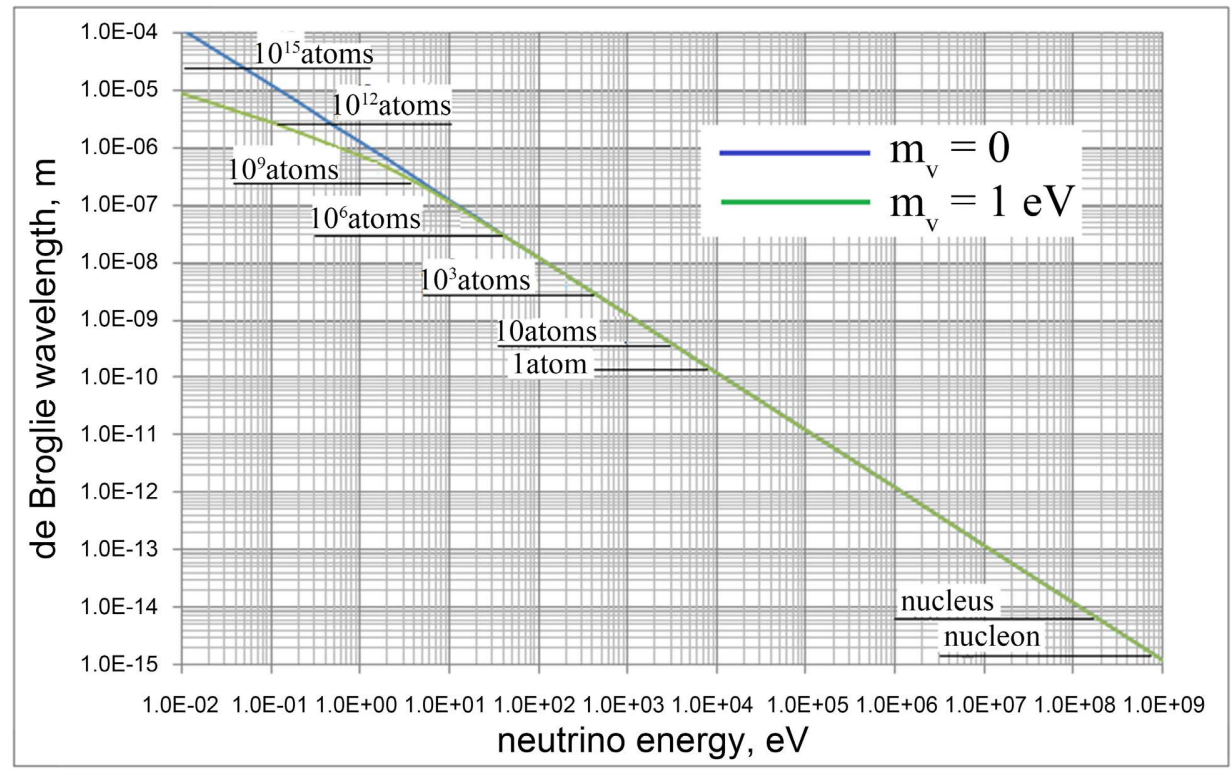

Figure 4. Dependence of de Broglie wavelength of neutrino or antineutrino (size of the interaction region) on the neutrino energy. Indicates how many atoms of solid or liquid matter are in the neutrino interaction region at a given energy.

Indeed, iron, cobalt and copper were found as impurities in nickel-hydrogen reactors that had been operated.

Appearance of iron from water in the Energoniva reactor [3] becomes clear. Combination of the nucleons contained in three water molecules and four electrons gives the iron nucleus:

$$
4 v^{\sim}+3 \mathrm{H}_{2} \mathrm{O}+4 \mathrm{e}^{-} \rightarrow{ }^{54} \mathrm{Fe}_{26}+87.81 \mathrm{MeV}
$$

Finally, it is possible to explain the paradoxical phenomenon discovered by Louis Kervran [34]: in the absence of calcium in chicken feed, hens managed to produce calcium from potassium:

$$
{ }^{39} \mathrm{~K}_{19}+{ }^{1} \mathrm{H}_{1} \rightarrow{ }^{40} \mathrm{Ca}_{20}+8.337 \mathrm{MeV}
$$

This transformation cannot occur through strong interaction channel due to "Coulomb barrier". And if it could, huge release of energy in this nuclear reaction would have incinerated the animal.

However, if the same transformation goes through the channel of weak interactions involving neutrinos, both of these problems are solved:

$$
v+{ }^{39} \mathrm{~K}_{19}+{ }^{1} \mathrm{H}_{1} \rightarrow{ }^{40} \mathrm{Ca}_{20}+v^{\prime}+8.337 \mathrm{MeV}
$$

Triggering this transformation low energy neutrinos can be generated during cellular metabolism. As a result of the nuclear reaction, two particles arise: calcium nuclei and neutrinos. Since in a system of two particles, energy is distributed inversely to mass, almost all the energy is carried away by neutrino, without leaving it in the chicken.

\section{Conclusions}

Unlike many other hypotheses, hypothesis of low-energy neutrinos participation 
in nuclear transformations allows us to explain many features of LENR:

- the appearance of a large variety of nuclides not only in "fuel", but also in surrounding matter;

- the need to heat or give matter particles energy in another way;

- the need for a sufficiently dense environment;

- no (or very low intensity) of hard nuclear radiation.

It is important to note that there is no "Coulomb barrier problem" in nuclear transformations involving neutrinos.

\section{Acknowledgements}

Authors would like to thank T. Timerbulatov, S. Zabavin and I. Stepanov for supporting the research and helping with equipment; R. Karabanov and I. Kamputin for high-quality analysis of chemical composition; N. Samsonenko, L. Urutskoev, A. Chistolinov and V. Vysotskiy for helpful discussions and valuable criticism; I. Savvatimova, A. Klimov, V. Zatelepin, R. Grinyer V. Zhigalov, G. Levi, A. Prosvirnov, S. Godin, V. Panchelyuga, A. Kovacs and many other people in Russia and abroad for their support and valuable advice.

\section{Conflicts of Interest}

The authors declare no conflicts of interest regarding the publication of this paper.

\section{References}

[1] Karabut, A.B., Kucherov, Y.R. and Savvatimova, I.B. (1992) Physics Letters A, 170, 265-276. https://doi.org/10.1016/0375-9601(92)90252-H

[2] Savvatimova, I.B. (2011) Journal of Condensed Matter Nuclear Science, 8, 1-19.

[3] Vachaev, A.V., Ivanov, N.I., Ivanov, A.N. and Pavlova, G.A. (1994) Method of Obtaining Elements and Device for Its Implementation. Patent of the Russian Federation No. 2096846, MKI G 21 G 1/00, H 05 H 1/24.

[4] Urutskoev, L.I., Liksonov, V.I. and Tsinoev, V.G. (2002) Annales de la Fondation Louis de Broglie, 27, 701-726.

[5] Ivoilov, N.G. (2006) Annales de la Fondation Louis de Broglie, 31, 115-123.

[6] Mastromatteo, U. (2016) Journal of Condensed Matter Nuclear Science, 19, 173-182.

[7] Biberian, J.-P. (2020) Transmutation Induced by Laser Irradiation. Le colloque RNBE 2020. 21 November.

[8] Parkhomov, A.G. and Karabanov, R.V. (2021) RENSIT, 13, 45-58. https://doi.org/10.17725/rensit.2021.13.045

[9] Parkhomov, A.G. and Karabanov, R.V. (2021) Journal of Forming Areas of Science, 27, 116-119. http://www.unconv-science.org/pdf/27/parkhomov2.pdf

[10] Parkhomov, A.G., Alabin, K.A., Andreev, S.N., et al. (2017) RENSIT, 9, 74-93. https://doi.org/10.17725/rensit.2017.09.074

[11] Parkhomov, A.G., Zhigalov, V.A., Zabavin, S.N, et al. (2019) Journal of Forming Areas of Science, 23-24, 57-63.

http://www.unconv-science.org/pdf/23/parkhomov2.pdf 
[12] Widom, A. and Larsen, L. (2006) The European Physical Journal C, 46, 107-111.

[13] Kuznetsov, V., Mishinsky, G., Penkov, F., Arbuzov, V. and Zhemenik, V. (2003) Annales de la Fondation Louis de Broglie, 28, 173-213.

[14] Filippov, D.V. and Urutskoev, L.I. (2004) Annales de la Fondation Louis de Broglie, 29, 1187-1206.

[15] Parkhomov, A.G. (2017) Journal of Forming Areas of Science, 17-18, 99-101.

[16] Parkhomov, A.G. (2018) International Journal of Unconventional Science, E3, 32-33. http://www.unconv-science.org/pdf/e3/parkhomov-en.pdf

[17] Martyushev, L.M. and Seleznev, V.D. (2006) Physics Reports, 426, 1-45. https://doi.org/10.1016/j.physrep.2005.12.001

[18] Lochak, G. (1985) IJTP, 24, 1019-1050. https://doi.org/10.1007/BF00670815

[19] Fredericks, K.A. (2013) Engineering Physics, 6, 15-45.

[20] Daviau, C., Fargue, D., Priem, D. and Racineux, G. (2013) Annales de la Fondation Louis de Broglie, 38, 139-153.

[21] Kovacs, A. (2020) Magnetic Monopole Mass and Charge Radius Calculation from Experimental Data Analysis. Proceedings of the 26th Russian Conference on Cold Nuclear Transmutations and Ball Lightning, Moscow, 28 September-2 October 2020, 205-219.

[22] Mishinsky, G.V. (2017) RENSIT, 9, 147-160. https://doi.org/10.17725/rensit.2017.09.147

[23] Dubovik, V.M., Shishkin, A.L., Baranov, V.A., Vinogradova, A.V. and Tatur, V.Yu. (2012) Investigation of the Characteristics of Magnetotroelectric Radiation Using Photofilm Detectors. http://www.trinitas.ru/rus/doc/0231/004a/02311041.htm

[24] Shoulders, K.R. (1987) EV: A Tale of Discovery. Jupiter Technologies, Austin.

[25] Parkhomov, A.G. (2019) International Journal of Unconventional Science, E4, 3-5. http://www.unconv-science.org/pdf/23/parkhomov1-en.pdf

[26] Cocco, A.G., Mangano, G. and Messina, M. (2007) Journal of Cosmology and Astroparticle Physics, 706, 15. https://doi.org/10.1088/1475-7516/2007/06/015

[27] Lazauskas, R., Vogel, P. and Volpe, C. (2007) Charged Current Cross Section for Massive Cosmological Neutrinos Impinging on Radioactive Nuclei. https://doi.org/10.1088/0954-3899/35/2/025001

[28] Parkhomov, A.G. (2018) Journal of Modern Physics, 9, 1617-1632. https://doi.org/10.4236/jmp.2018.98101

[29] Parkhomov, A.G. (2019) Space. Earth. Human. New Views on Science. 251 p. https://www.amazon.com/dp/171122121X

[30] KATRIN Collaboration (2022) Nature Physics, 18, 160-166. https://doi.org/10.1038/s41567-021-01463-1

[31] Thomas, S.A., Abdalla, F.B. and Lahav, O. (2010) Physical Review Letters, 105, Article ID: 03130. https://doi.org/10.1103/PhysRevLett.105.031301

[32] Kajita, T. (2015, December 8) Discovery of Atmospheric Neutrino Oscillations. Nobel Lecture. https://www.nobelprize.org/uploads/2018/06/kajita-lecture.pdf

[33] Aharonov, Y. and Avignore, F.T. (1987) Physical Review Letters, 58, 1173-1175. https://doi.org/10.1103/PhysRevLett.58.1173

[34] Kervran, L. (1998) Biological Transmutations. Happiness Press, Magalia. 